\title{
ANATOMICAL VARIATIONS OF SUPERFICIAL VEINS PATTERN IN CUBITAL FOSSA AMONG NORTH WEST ETHIOPIANS
}

\author{
Abebe Ayalew Bekel ${ }^{1}$ (MSc.), Assegedech Bekele Bekalu² (PhD), Abebe Muche Moges $^{2}$ (PhD), Mueez Abraha \\ Gebretsadik $^{2}$ (MSc.)
}

1: Bahir Dar University College of Medicine and Health Sciences

2: Gondar University College of Medicine and Health Sciences

Correspondence to Abebe Ayalew Bekel Department of Human Anatomy, College of Medicine and Health Sciences Bahir Dar University P. O. Box: 79, Bahir Dar, Ethiopia. Email: abe3a16b@gmail.com Telephone: +251918040350, Fax: 0582205932

\begin{abstract}
Superficial veins in the cubital fossa are a common site for obtaining venous blood for analysis, transfusion, and intravenous therapy. These superficial veins are often visible through the skin, and are anatomically variable. These include cephalic vein, basilic vein, median cubital vein, and median antebrachial vein. The objective of this study is to assess variations of superficial veins arrangement in the cubital fossa. A tourniquet was applied $10 \mathrm{~cm}$ proximal to elbow crease for about three minutes with active flexion and extension of fingers until the veins are exposed for observation. Four types of superficial venous patterns were identified in cubital fossa. From the total of 800 studied arms 58.5\%, $18.6 \%, 14 \%, 8.9 \%$ had type 1 , type 2 , type 3 , and type 4 patterns, respectively. In the majority of studied subjects, the veins patterns go with the findings of former studies. However, some rare venous patterns were also identified.
\end{abstract}

Key words: Cubital fossa; superficial veins; variations

\section{INTRODUCTION}

The main superficial veins in the cubital fossa are cephalic, basilic and median veins (median cephalic, median basilic, median cubital and median antebrachial). These superficial veins are very often visible through the skin (Vasuda, 2013; Azhar et al., 2014; Ukoha et al., 2013). The cephalic vein ascends in the subcutaneous tissue from the lateral aspect of dorsal venous network, proceeding along the lateral border of the wrist and anterolateral surface of the proximal forearm and arm. Anterior to the elbow, it communicates with medial cubital vein, which passes obliquely across the anterior aspect of the elbow in the cubital fossa and joins basilic vein.
It courses superiorly along deltopectoral groove and enter to clavipectoral triangle. It then pierces costocoracoid membrane and part of clavipectoral fascia, joining the terminal part of the axillary vein (Vasuda, 2013; Moore and Dalley, 2010; Kiray et al., 2013).

The basilic vein ascends in subcutaneous tissue from medial end of the dorsal venous network along medial side of the forearm and inferior part of the arm. It then passes deeply near the junction of the middle and inferior thirds of the arm, piercing brachial fascia and running superiorly parallel to brachial artery and medial cutaneous nerve of the forearm to the axilla, 
where it merges with the accompanying veins of the axillary artery to form the axillary vein (Vasuda, 2013; Moore and Dalley, 2010; Syed et al., 2011; Jose et al., 2003; Naveen et al., 2012; Shenoy, 2013).

The medial cubital vein is the branch that connects the cephalic vein to the basilic vein. It usually shunts some of the blood collected by the cephalic vein to the basilic vein $(70-80 \%$ of individuals) (Vasuda, 2013; Moore and Dalley, 2010). The median antebrachial vein is highly variable. It begins at the base of the dorsum of the thumb, curves around the lateral side of the wrist, and ascends in the middle of the anterior aspect of the forearm between cephalic and basilic veins. It sometimes divides into median basilic and median cephalic veins, which joins basilic and cephalic veins, respectively (Moore and Dalley, 2010; Ukoha et al., 2013).

Regardless of their pattern variability in approximately $20 \%$ of people (Moore and Dalley, 2010), venipuncture using superficial veins in the cubital fossa for obtaining a blood sample is one of the most common procedures in the emergency room. It is performed by various medical practitioners such as physician, nurse, emergency medical technician. However, because of anatomical variations of superficial veins, venipuncture procedure can be most frustrating task especially for the inexperienced health workers. It will be worst in shocked patients as the vessels are collapsed and difficult to visualize. Improper management of the procedure may result in injuries such as bruising, hematoma, and sensory change in the region. In order to implement safe way of venipuncture and save the precious life of patients, understanding the pattern of superficial veins anatomy in general and the cubital fossa in particular is mandatory (Hyunsu et al., 2015). Studies done in different part of the world showed that different varieties of superficial veins arrangement in the cubital fossa. Hence, this study attempted to identify the different patterns of superficial veins, prevalence of each variant in Ethiopia and compared patterns of superficial veins in right and left cubital fossa and access the result to health professionals, anatomists, students of medicine and health sciences.

\section{MATERIALS AND METHODS}

The pattern of superficial venous arrangement in the cubital fossa of 400 randomly selected subjects in North West Ethiopia were noted. After informed verbal consent was taken from participants, data was gathered by the researcher based on observation after exposing both upper limbs distal to half of the arm and extending elbow joint in supine position. Then tourniquet was applied $10 \mathrm{~cm}$ proximal to elbow crease for about three minutes with active flexion and extension of fingers until the veins are exposed for observation. The quality of data was ensured by close observation when superficial cubital veins were exposed after tourniquet was applied and recorded the observed arrangement on the corresponding data collection format and captured the pattern of the veins by digital camera before removal of the tourniquet. The data were entered and coded into SPSS version 16. Descriptive statistical analysis was performed. Chi-Square test was used to assess the association of superficial venous pattern in cubital fossa with body side. $\mathrm{P}<0.05$ was considered as statistical significance.

\section{RESULTS}

A total of 400 subjects in age range of 18 to 30 years (mean age, 21.8 and standard deviation, 2.0) comprising $326(81.5 \%)$ males and 74
(18.5\%) females were assessed for superficial venous arrangements in cubital fossa. In this study, four types of superficial venous 
arrangements were identified (Table 1). Type1 (Table 1, Figure 1) pattern was seen in 468 $(58.5 \%)$ cubital fossae (Table 2). It was observed in $243(30.4 \%)$ and $225(28.1 \%)$ right and left cubital fossa, respectively. Besides, it was identified in $361(55.4 \%)$ males' and 107 (72.3\%) females' cubital fossae (Table 3). Type 2 (Table 1, Figure 2) was observed in 149 $(18.6 \%)$ cubital fossae (Table 2$)$. Sixty five $(8.1 \%)$ right and $84(10.5 \%)$ left cubital fossae had this type of arrangement. It was also noticed in $118(18.1 \%)$ and $31(20.9 \%)$ males $^{\prime}$ and females' cubital fossae, respectively (Table 3 ).
Type 3 (Table 1, Figure 3) pattern was detected in $112(14 \%)$ cubital fossae (Table 2). Fifty two $(6.5 \%)$ right and $60(7.5 \%)$ left cubital fossae had such kind of superficial venous arrangement. One hundred six (16.3\%) males' and $6(4 \%)$ females' cubital fossae had type 3 pattern (Table 3). Type 4 (Table1, Figure 4) variant was noticed in $71(8.9 \%)$ cubital fossae (Table 2). It was detected in 41 (5.2\%) right and $30(3.7 \%)$ left cubital fossae. Sixty seven $(10.3 \%)$ males' and $4(2.7 \%)$ females' cubital fossae had this type of superficial venous arrangement (Table 3).

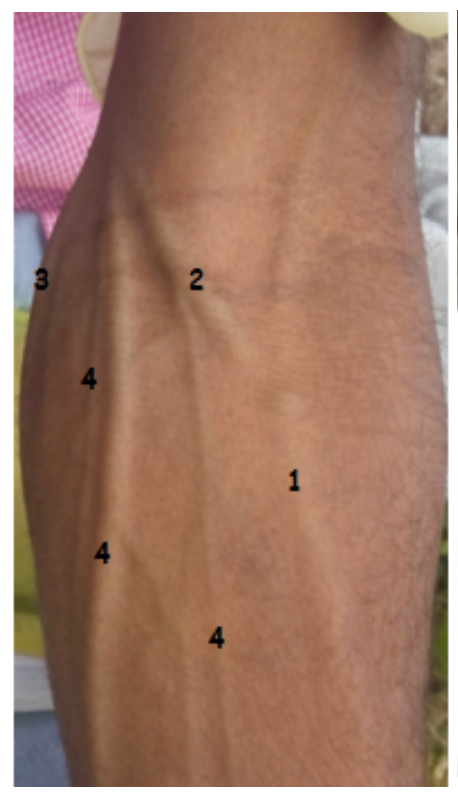

Figure 1: A Photograph showing type 1 arrangement of superficial veins in left cubital fossa. 1: Cephalic Vein; 2: Median Cubital Vein; 3: Basilic Vein; 4: Tributaries to Median Cubital Vein

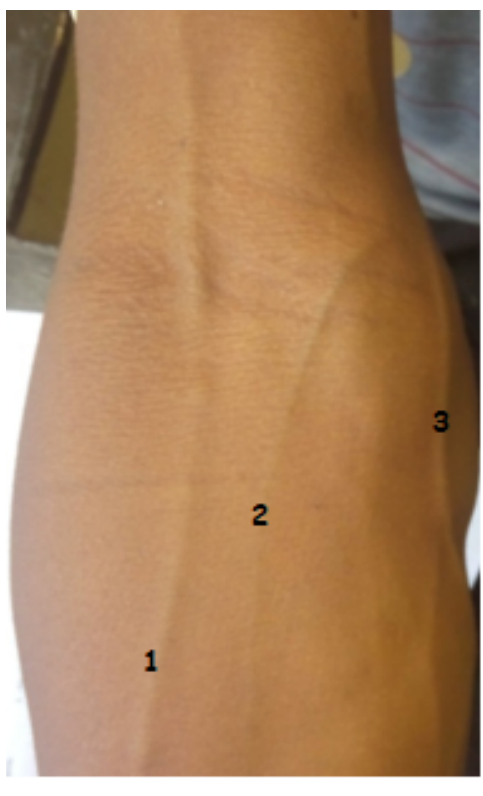

Figure 2: A Photograph shows type 2 pattern of superficial veins in right cubital fossa. 1: Cephalic Vein; 2: Median Antebrachial Vein; 3: Basilic Vein

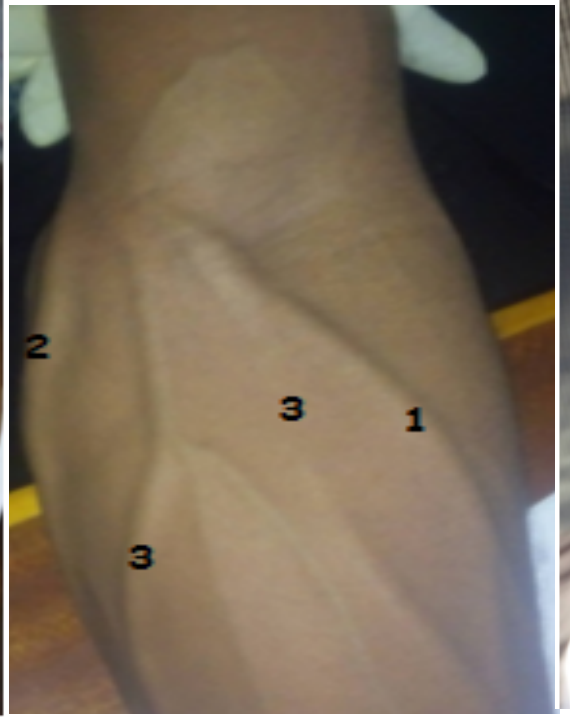

Figure 3: A Photograph shows type 3 arrangement of superficial veins in left cubital fossa. 1: Cephalic Vein; 2: Basilic Vein; 3: Tributaries to Cephalic Vein

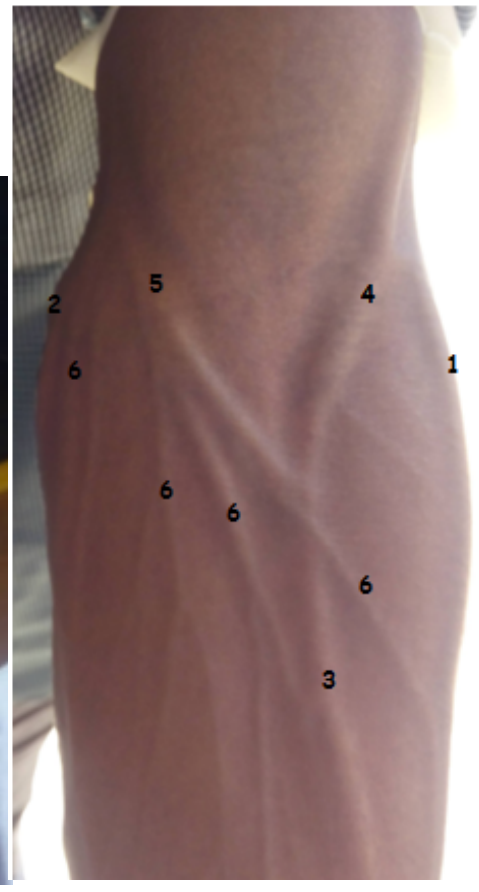

Figure 4: A Photograph showing type 4 arrangement of superficial veins in left cubital fossa. 1: Cephalic Vein; 2: Basilic Vein; 3: Median Antebrachial Vein; 4: Median Cephalic vein; 5: Median Basilic Vein; 6: Other tributaries 
Table 1: Types of superficial veins pattern in cubital fossa

\begin{tabular}{|l|l|}
\hline Types & Description \\
\hline Type 1 & $\begin{array}{l}\text { Median cubital vein arise from cephalic vein a few centimeters below elbow joint and } \\
\text { passes obliquely upward to medial side to join basilic vein a few centimeters above } \\
\text { elbow joint and receives tributaries from the front of the forearm. }\end{array}$ \\
\hline Type 2 & $\begin{array}{l}\text { Median antebrachial vein joins basilic vein without establishment of communication } \\
\text { between cephalic and basilic vein. }\end{array}$ \\
\hline Type 3 & $\begin{array}{l}\text { Cephalic vein runs from lateral to medial where it joins basilic vein and receives } \\
\text { tributaries from the front of the forearm; no proximal cephalic vein. }\end{array}$ \\
\hline Type 4 & $\begin{array}{l}\text { Median antebrachial vein divides into median cephalic and median basilic veins in } \\
\text { cubital fossa which joins cephalic and basilic veins, respectively. }\end{array}$ \\
\hline
\end{tabular}

Generally, it was observed that out of 400 study participants, $319(80 \%)$ of them had the same type of pattern in both arms while the remaining $81(20 \%)$ had different patterns in each cubital fossa. However, there was no statistically significant association between body side (right and left cubital fossae) and superficial veins arrangement in cubital fossa ( $P$ value $=0.412$ ) (Table 2). The present study clearly indicated the prevalence of type 1 and 2 superficial venous arrangements in cubital fossa are higher in females than males. The reverse holds true for type 3 and 4 superficial venous arrangements, which are prevalent in cubital fossae of male study participants. Hence, there was statistically significant association between sex and superficial veins arrangement in cubital fossae ( $P$ value $=0.001)($ Table 3$)$.

Table 2: Patterns of superficial veins in right and left cubital fossa

\begin{tabular}{|c|l|l|l|l|l|l|l|}
\hline \multicolumn{2}{|c|}{} & \multicolumn{6}{l}{ Patterns of superficial veins } \\
\cline { 2 - 7 } & Type 1 & Type 2 & Type 3 & Type 4 & Total & P value \\
\cline { 2 - 7 } & Number (\%) & $\begin{array}{l}\text { Number } \\
(\%)\end{array}$ & $\begin{array}{l}\text { Number } \\
(\%)\end{array}$ & $\begin{array}{l}\text { Number } \\
(\%)\end{array}$ & $\begin{array}{l}\text { Number } \\
(\%)\end{array}$ & 0.412 \\
\hline \multirow{3}{*}{ Body side } & Right & $243(60.7)$ & $65(16.3)$ & $52(13)$ & $40(10)$ & $400(100)$ & \\
\cline { 2 - 7 } & Left & $225(56.2)$ & $84(21)$ & $60(15)$ & $31(7.8)$ & $400(100)$ & \\
\hline \multicolumn{2}{|c|}{ Total } & $468(58.5)$ & $149(18.6)$ & $112(14)$ & $71(8.9)$ & $800(100)$ & \\
\hline
\end{tabular}

Table 1: Distribution of superficial cubital venous types for both sexes

\begin{tabular}{|c|c|c|c|c|c|c|c|}
\hline & \multicolumn{6}{|c|}{ Patterns of superficial veins } \\
\hline & & Type 1 & Type 2 & Type 3 & Type 4 & Total & $P$ value \\
\hline & & $\begin{array}{l}\text { Number } \\
(\%)\end{array}$ & $\begin{array}{l}\text { Number } \\
(\%)\end{array}$ & $\begin{array}{l}\text { Number } \\
(\%)\end{array}$ & $\begin{array}{l}\text { Number } \\
(\%)\end{array}$ & $\begin{array}{l}\text { Number } \\
(\%)\end{array}$ & \multirow[t]{4}{*}{0.001} \\
\hline \multirow[b]{2}{*}{ Sex } & Male & $361(55.4)$ & $118(18.1)$ & $106(16.2)$ & $67(10.3)$ & $652(100)$ & \\
\hline & Female & $107(72.3)$ & $31(20.9)$ & $6(4.1)$ & $4(2.7)$ & $148(100$ & \\
\hline & Total & $468(58.5)$ & $149(18.6)$ & $112(14)$ & $71(8.9)$ & $800(100)$ & \\
\hline
\end{tabular}




\section{DISCUSSION}

Variations in the superficial veins of upper limb are well known. In embryonic life the veins arise from capillary plexus, which is increased by sprouting and anatomizing and then fuse, enlarge forming fewer and large channels. Genetic and hydrodynamic factors play an important role in the final pattern of veins, which may result in variations (Vasuda, 2013).

Most health professionals are aware of only two patterns of superficial venous arrangement in the cubital fossa as it was described in the standard textbooks of anatomy. In the first pattern the cephalic vein gives of the median cubital vein which pass upward and medially to join the basilic vein (Moore and Dalley, 2010). In the present study, it was the commonest arrangement of superficial veins in about $59 \%$ of cubital fossa. Our finding was in line with reports in Jordanians (48.5\%) (Faraj and Eman, 2014), Malaysians (68\%) (Dharap and Shaharuddin, 1994) and Koreans (50.1\%) (Hyunsu et al., 2015). However, the prevalence was relatively low in Nigerians (28\%) (Ukoha et al., 2013) and Malaysians (33.7\%) (Azhar et al., 2014) but extremely high in Indians (94.4\%) (Vasuda, 2013).

The second variant depicted in standard anatomy books is formed of median antebrachial vein which ascends in anterior part the forearm and divided into median cephalic and median basilic veins which join cephalic and basilic veins, respectively. This group has a general incidence of $8.9 \%$ and it is the fourth variant in the present study (Table 2). Similarly, the prevalence of this venous pattern was low in researches conduced in Jordanians (Faraj and Eman, 2014) Malaysians (16\%) (Dharap and Shaharuddin, 1994) and Indians (2.4\%) (Vasuda, 2013). However, this variant was reported as the commonest form of venous arrangement in Nigerians (33\%) (Ukoha et al., 2013), Malaysians (26\%) (Azhar et al., 2014) and Koreans (47\%) (Hyunsu et al., 2015).

In the present study, two other arrangements of superficial veins were detected in the cubital fossa, in which many health professionals may not be familiarize. Type 2 pattern is formed of median antebrachial vein joins basilic vein without establishment of communication between cephalic and basilic veins and was seen in $18.6 \%$ of the study arms. This pattern was also reported in Malaysians (5.6\%) (Azhar et al., 2014) and Japanese with unknown prevalence (Anant et al., 2007; Mikuni et eal., 2013) However, it was not detected in studies conducted (Vasuda, 2013; Ukoha et al., 2013; Faraj and Eman, 2014; Dharap and Shaharuddin, 1994; Hyunsu et al., 2015)

In type 3 , cephalic runs from lateral to medial where it joins basilic vein and receives tributaries from the front of the forearm; no proximal cephalic vein and it was examined in about $14 \%$ of the study subjects. However, the incidence of type 3 venous arrangement was high in Nigerians (25\%) (Ukoha et al., 2013) but low in Malaysians (4.7\%) (Dharap and Shaharuddin, 1994) and Indians (0.8\%) (Vasuda, 2013).

Interestingly, out of 400 study subjects, about $80 \%$ had the same type of pattern in both arms while the remaining $20 \%$ had different patterns in each arm. However, there was no statistically significant association between body side (right and left cubital fossa) and superficial veins arrangement in cubital fossa ( $P$ value $=0.412$ ) (Table 2). ). It was supported by various studies conducted in Malaysia (Azhar et al., 2014), Jordan (Faraj and Eman, 2014) and Korea (Hyunsu et al., 2015). On the other hand, a study done in Nigerians (Ukoha et al., 2013) revealed that out of 135 studied subjects 100 $(74.1 \%)$ have different patterns on the right and left side of cubital fossa.

Of note, the present study clearly indicated higher prevalence of type 1 and 2 superficial venous arrangements in cubital fossa of females than males. The reverse holds true for type 3 and 4 superficial venous arrangement, which are prevalent in cubital fossae of male study participants. Hence, there was statistically significant association between sex and 
superficial veins arrangement in cubital fossae ( $P$ value $=0.001)$ (Table 3$)$. Our finding fits well with studies done in Jordanians (Faraj and Eman, 2014) and Nigerians (Ukoha et al., 2013). However, in studies conducted in Malaysians (Azhar et al., 2014; Dharap and Shaharuddin, 1994;), Indians (Vasuda, 2013) and Koreans (Hyunsu et al., 2015) sex had no influence on the distribution of cubital superficial veins.

In conclusion, results from this study illustrated that superficial venous arrangement in the cubital fossa of study subjects were grouped into four distinct types with the prevalence of $58.5 \%$

Conflict of interest: All authors of the paper have no conflict of interest. The research was not funded. (type 1), 18.6\% (type 2), 14\% (type 3), and $8.9 \%$ (type 4 ). It was also found that sex influence was significant on superficial venous pattern of the cubital region. But the right and left side difference was not statistically significant. The study of the arrangement of superficial veins in the cubital fossa in our environment is vital to aware their patterns and prevalence, which would be very useful to for health professionals who approaches these superficial veins, especially under emergency conditions.

\section{REFERENCES}

1. Anant K, Manish S, Nitin G et al. 2007. Radio-median cubital / radiocephalic arteriovenous fistula at elbow to prevent vascular steal syndrome associated with brachiocephalic fistula. Indian J Urol; 23: 261-4.

2. Azhar H, Saravanan R, Azreen S, Amer H. 2014. Pattern of superficial venous of the cubital fossa among volunteers in a tertiary hospital. Trop Med Surg; 2: 1-4.

3. Dharap AS, Shaharuddin MY. 1994. Patterns of superficial veins of the cubital fossa in Malaysians. Med J Malaysia; 49: 239-41.

4. Faraj A, Eman R.2014. Pattern of superficial venous arrangement in the cubital fossa of adult Jordanians. J Med J; 48: 269-74.

5. Hyunsu L, Sang L, Sung K, Woo C. 2015. Variations of the cubital superficial vein investigated by using the intravenous illuminator. Anat Cell Biol; 48: 62-5.

6. Jose C, Costa B, Serafim V, Andre L, Emil B. 2003. Anatomy of the basilic vein in the arm and its importance in surgery. Braz J Morphol Sci; 20: 171-5.

7. Kiray A, Erqur I, Tayefi H, Baqriyanik H, Bacakoglu A. 2013. Anatomical evaluation of the superficial seins of the upper extremity as graft donor source in microvascular reconstructions: $A$ cadaveric study. Acta Orthop Traumatol Turc; 47: 405-10.

8. Mikuni Y, Chiba S, Tonosaki Y. 2013. Topographical anatomy of superficial veins, cutaneous nerves, and arteries at venipuncture sites in the cubital fossa. Anat Sci Int ; 88: 46-57.

9. Moore K, Dalley A. 2010. Clinically Oriented Anatomy, $7^{\text {th }}$ ed. London: Lippincott and Wlikins, : 1407

10. Naveen K, Ashwini P, Mohandas R, Sateesha B. 2012. The venous chiasma between the basilic vein and the brachial vein. J Clin Diagn Res; 6:1539-40.

11. Shenoy S. 2009. Surgical anatomy of upper arm. J Vas Access; 10: 223-32.

12. Syed O, Ismail N, Younes $\mathrm{H}$ et al. 2011. Prevalence of variant brachial-basilic vein anatomy and implications for vascular access planning. J Vas surg; 53: 720-4.

13. Ukoha U, Oranusi C, Okafor J, Ogugua P, Obiaduo A. 2013. Patterns of superficial venous arrangement in the cubital fossa of adult Nigerians. Niger J Clin P; 16: 104-9.

14. Vasuda T. A 2013. study on superficial veins of upper limb. NJCA; 2: 204-8. 